\title{
INFLAMMATORY COMPLICATIONS OF CANCER OF THE CAECUM AND ASCENDING COLON
}

\author{
By M. R. EwING, F.R.C.S. (Eng. and Ed.) \\ From the Department of Surgery, Postgraduate Medical School of London
}

There occurs in the right lower abdomen a wide variety of pathological lesions many of which present clinically as a lump. Chief among them are carcinoma and the 'appendix mass' or the appendix abscess. Recent experience has brought home to me how confused the diagnosis may be between these two; for not only may they be very much alike when presenting alone, but still more perplexing is the occurrence of the two together, as is so often the case. Clinicians of riper experience than mine will doubtless number in recollection many patients presenting, in their management, just the problems which I shall presently describe. It is also true that there have been several contributions on this aspect of caecal cancer in the literature of the past 20 years or so (Wakeley and Rutherford, r932; Bartlett and Miller, I940; Mayo, 194I; Brown, Colvert and Brush, 1948). My only excuse in 'rediscovering ' what is already known to a few, is the somewhat disturbing lack of awareness (or so at least it seems to me) of the high incidence of the inflammatory complications of right colon cancer, a deficiency which many of the standard textbooks of surgery do little or nothing to make good.

\section{Pathogenesis}

Cancer in the proximal colon is often complicated by inflammatory disease, and this in a variety of ways:-

1. The tumour extends through the whole thickness of the bowel wall and breaks down to allow intestinal contents to leak out into the cellular tissues beyond (Fig. Ia).

This is probably the commonest happening and is especially a -feature of the typical proliferating tumours of the proximal colon.

Even when the tumour has not penetrated the whole thickness of the bowel wall (either naked eye or on microscopy) infection may still find its way out into the surrounding connective tissue (Fig. Ib). Microscopic evidence of such leakage is commonly encountered by the pathologist either in the form of an acute inflammatory reaction or even more strikingly, by the presence of vegetable material surrounded by a foreign-body giant cell response. Even when the infection is more advanced it may still be unsuspected clinically and overlooked at operation. It may be first discovered during the gross dissection of the pathological specimen as a small abscess cavity plastered up against the wall of the bowel at the site of the cancer.

If the abscess is bigger and more obvious it will be encountered at operation as a disturbing complication:-

(a) Because it obscures the diagnosis.

(b) Because it increases the technical difficulties of the resection, e.g. in recognition and avoidance of the ureter.

(c) Because it often makes it exceedingly diffcult to define and to recognize the limits of extension of the malignant disease.

(d) It may lead to the death of the patient when the disease is still resectable and curable, and even with survival its presence increases considerably the likelihood of post-operative morbidity.

(e) It may delay resection of the tumour and add a further stage to what may already be planned as a multi-stage procedure.

As an offset to these very considerable disadvantages, the lucky warning signal of an acute infective complication may sometimes serve to draw attention early to a silent and previously unnoticed growth.

If the inflammatory process is still more in evidence it may obscure completely at operation the associated underlying and causative new growth. Not only will the presence of the tumour readily be overlooked at operation, but the inflammatory complications will often dominate the clinical picture to confuse and confound the unwary and unexperienced.

2. Carcinoma of the caecum may co-exist with acute appendicitis, the relationship between the 


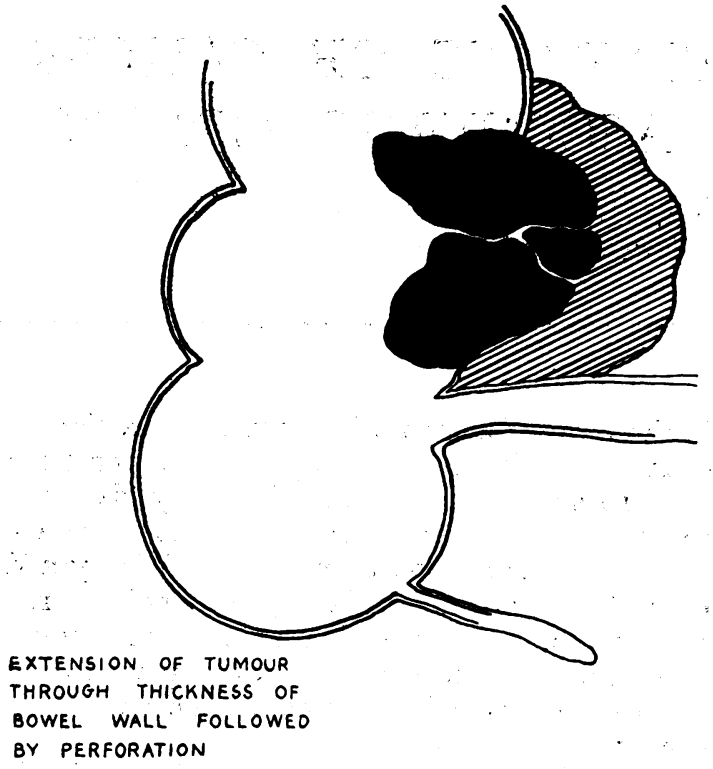

Fig. If

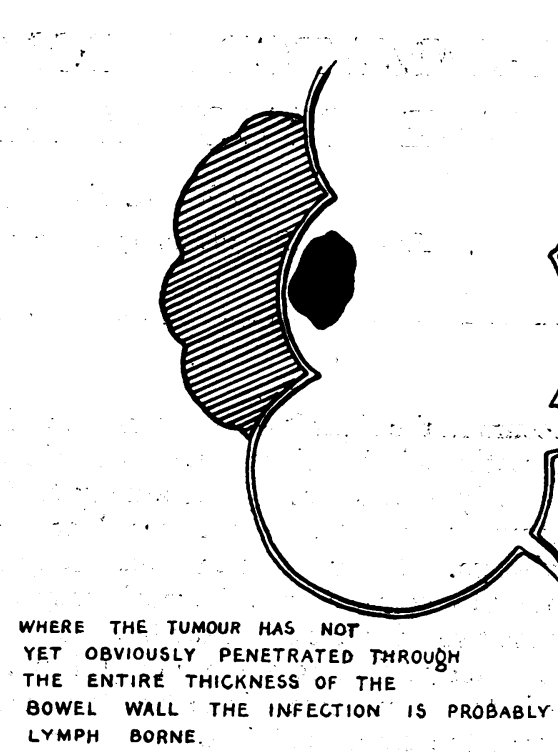

FIG. Ib



FIg. IC

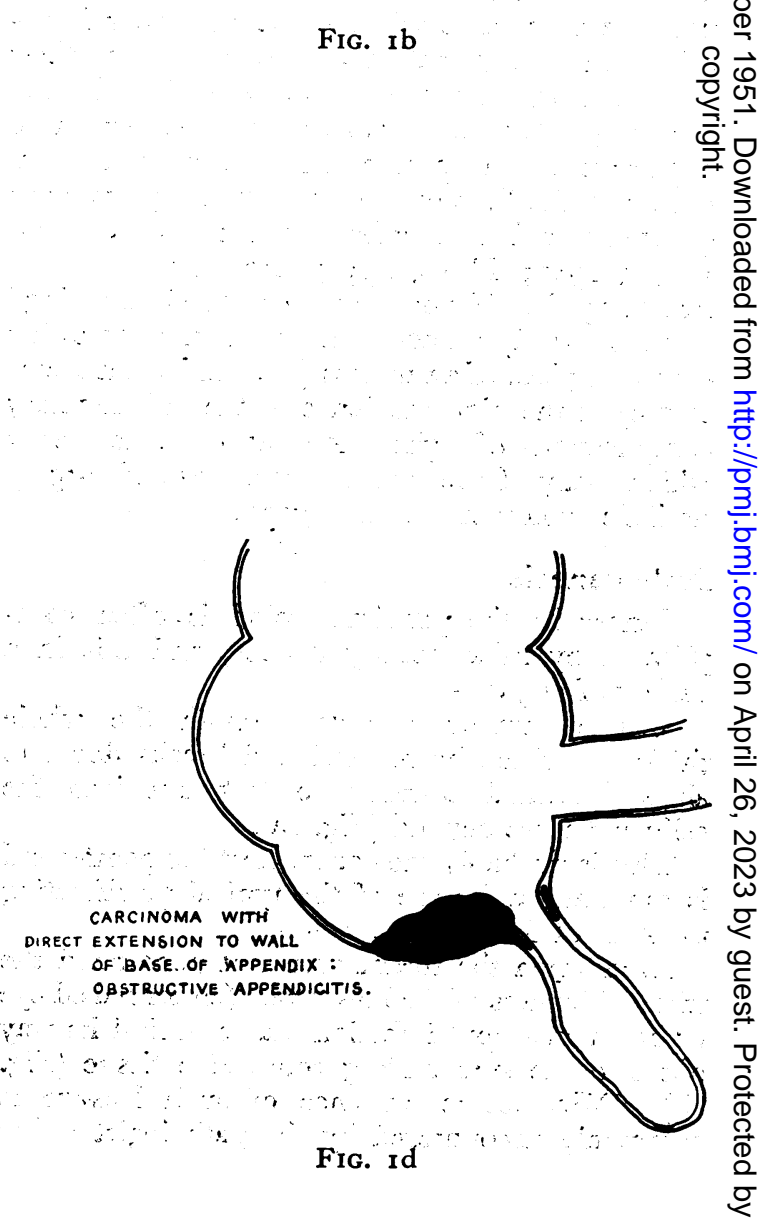


two being apparently one of cause and effect. 'The acute inflammation in the appendix can be precipitated in one of two ways.

(a) Commonly there is blockage of the lumen, usually by a bulky proliferating tumour, which grows over and obscures completely the appendicular orifice (Fig. Ic). Less frequently there is direct extension of the malignant tissue into the wall of the appendix at its base, with effective narrowing and final complete blockage of the lumen (Figs. Id and 2).

Whatever the mechanism of the obstruction, the result is the same-as it almost invariably is whenever the lumen of the appendix is blocked by whatever cause-namely, the development of acute obstructive appendicitis, which is no different in its behaviour from that due to more benign



FIG. 2.-Section through base of appendix to show diffuse infiltration of its wall by growth ( $\times 15)$.

causes. Gangrene and perforation occur early, with widespread peritonitis or local abscess formation as common sequelae. It will be appreciated that, at this stage, the symptoms of the complicating acute obstructive appendicitis must often overshadow those of the less urgent caecal neoplasm.

(b) When a growth blocks the colon some way distal to the ileo-caecal valve, the caecum becomes progressively more distended. Under such conditions it seems highly probable that the appendix too will share in the distension and will find increasing difficulty in emptying its content into the caecum. It may well be that under such conditions what we might reasonably call 'stercoral appendicitis ' may result (Fig. 3a). I must, however, admit that this conception is largely hypothetical and I can quote from my experience no single instance of such a happening.

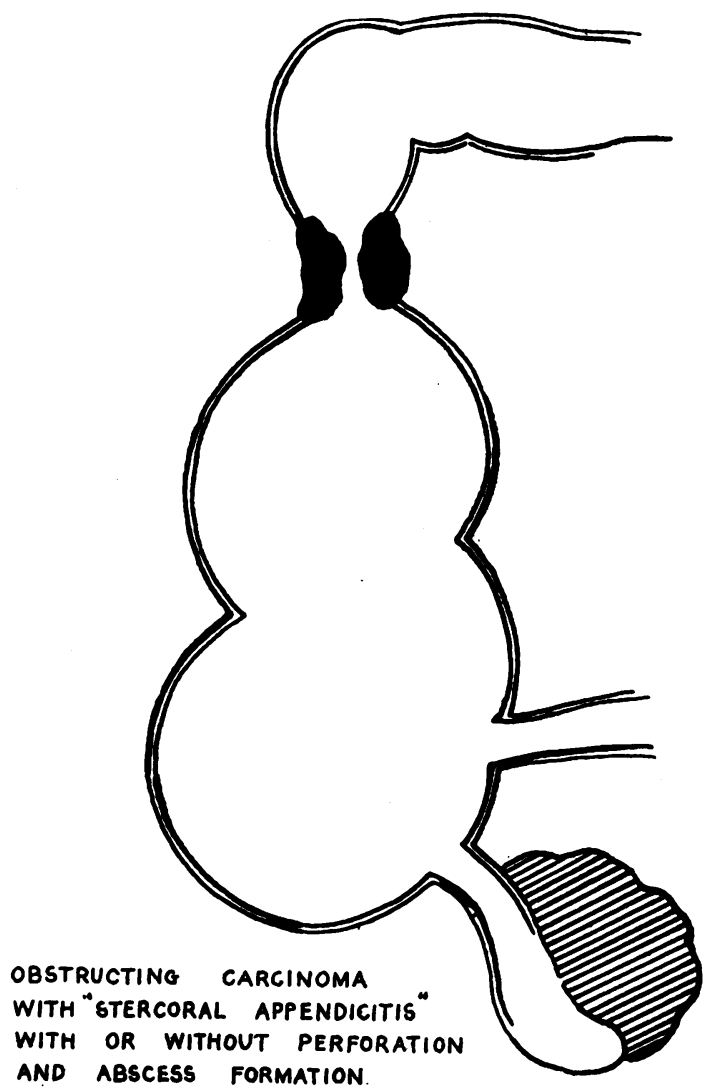

FIG. 3a

3. Complete intestinal obstruction in the large bowel at any level distal to the caecum leads to distension of the whole length of the colon proximal to the obstruction; it is the caecum, however, which suffers most. Of the gross caecal distension under such conditions the surgeon is well aware; the risk at operation of rupturing the overstretched, paper-thin, necrotic caecal wall is always in his mind when he explores the abdomen in the presence of a neglected large bowel obstruction. Spontaneous rupture of a stercoral caecal ulcer is a calamity of the first order, a rapidly spreading and very lethal peritonitis being the almost invariable sequel (Fig. 3b). Sometimes, happily, its perforation may occur more insidiously to give time for the development of an encapsulated pericolic abscess, but this is not often the case.

\section{Frequency}

I was recently impressed by a succession of patients with cancer of the right half of the colon, in each of whom the clinical picture was dominated by a complicating infection. On going through the records of Hammersmith Hospital for the years 


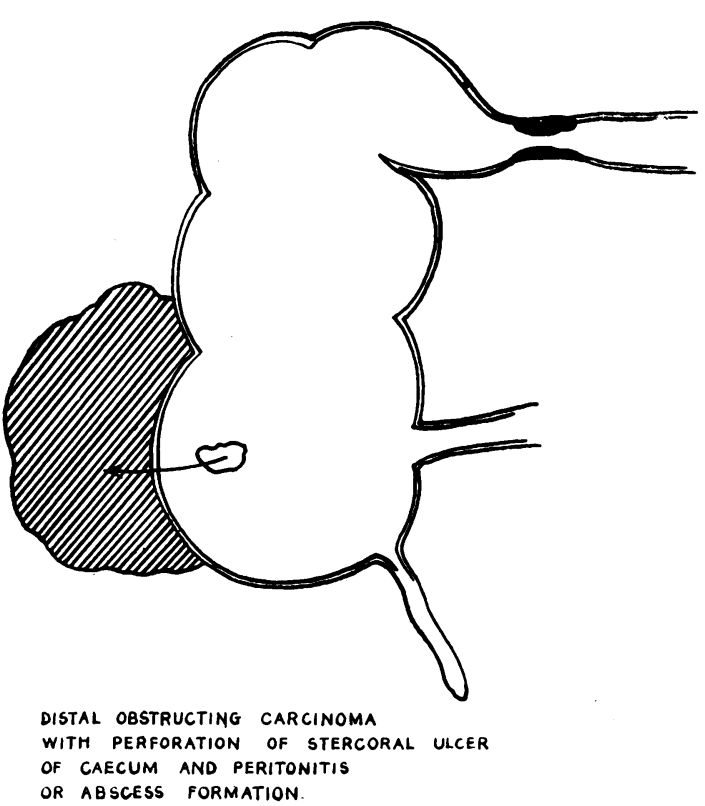

FIG. $3 b$

I 939 to I949, I have been able to collect 42 cases of carcinoma of the caecum, ascending colon, and hepatic flexure. In no less than 17 of the 42 there was evidence of infection, and in the great majority of these of sufficient seriousness to alter the clinical picture in varying degree.

\section{Effect on Clinical Picture}

The presence of infection affects the symptoms and signs in these patients in a variety of ways, some of which I propose now briefly to discuss with illustrations from my own experience and from our hospital records.

(a) The obvious case of cancer of the large bowel, where the incident of inflammation leads to some uncertainty in the diagnosis or requires the addition of a further step-that of drainage of a pericolic abscess-to what may already, in an obstructed case, be a time-consuming staged procedure.

Case I. A pale, worried old man was admitted with a large lump in the right iliac fossa diagnosed as a carcinoma of the caecum. His temperature on the evening of admission rose to $\mathrm{IOI}^{\circ}$ and continued during the next few days to swing between $99^{\circ}$ and $101^{\circ}$, while the abdominal tumour increased steadily in size and became acutely tender. On the fifth day after admission an ileo-transverse colostomy was fashioned and through a second lateral muscle-cutting incision $500 \mathrm{ml}$. of foul pus were evacuated from a large abscess cavity lying lateral to the caecum. When hemicolectomy was c arried out about one month later, a large cauli-

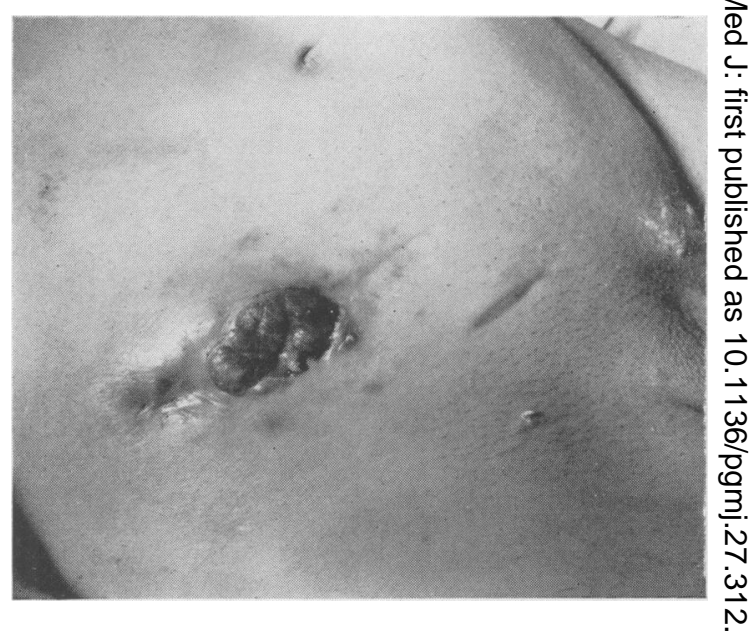

FIG. 4.-Case 10. Clinical diagnosis acute appendicitis. $\frac{G}{G}$ Found at operation to have a perforated appendix with abscess formation. Cancer of caecum overlooked until it erupted on to skin surface through the unhealed operation wound.

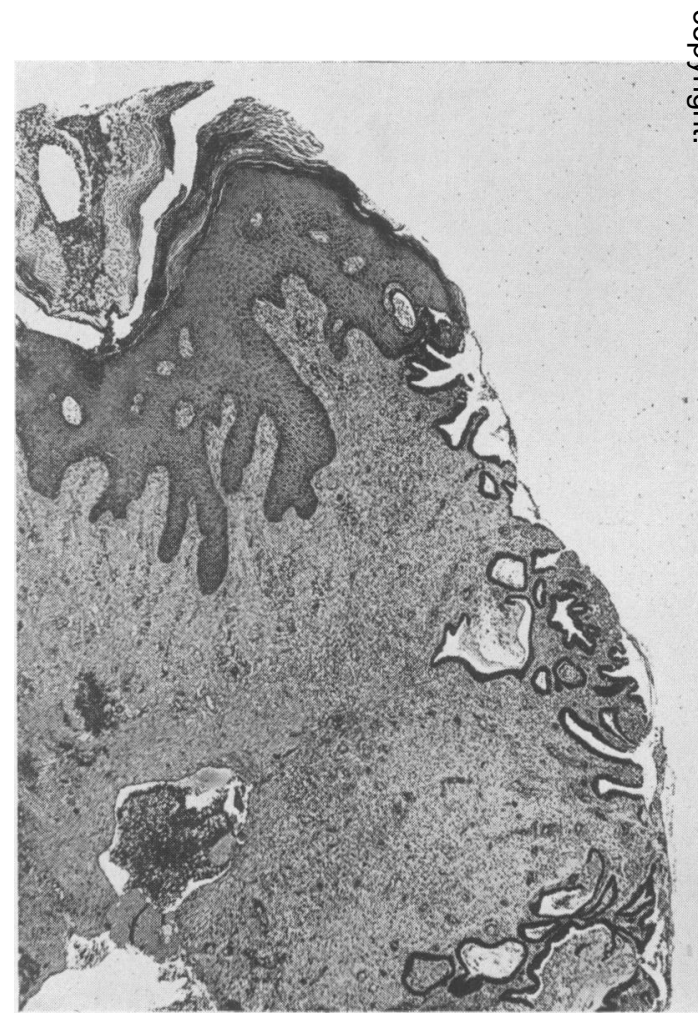

FIG. 5.-Case ro. Section taken from the margin of the sinus shown in Fig. 4. The extension of the adenocarcinoma to the skin is clearly shown $(\times 39)$. 
flower growth was found obstructing the ileocaecal valve and the appendix. There was an associated massive honeycomb abscess extending widely into the iliacus muscle.

(b) The patient in whom the shortness of the history and of the presenting symptoms suggests a diagnosis of acute affendicitis or of appendix abscess, but where, often as a comflete surprise, a colon cancer is discovered at laparotomy.

At operation there may be found either acute appendicitis or a pericaecal abscess or neither, which, matters not at all; what is really important is the recognition at this stage of the primary pathology, which has been earlier overlooked at the bedside.

Case 2. A plethoric old gentleman of $7 \mathrm{I}$ years was admitted to hospital as an emergency. For about a week he had complained of spasms of violent umbilical colic and had vomited once.

His abdomen was uniformly distended and there was tenderness and rigidity in the right iliac fossa. A lump rising out of the pelvis slightly to the right of the midline was thought to be a distended bladder, but it did not disappear after catheterization. His temperature was $101^{\circ}$. A diagnosis of acute appendicitis was made and expectant treatment advised. By the following afternoon, however, the distension had become much more obvious and a 'scout' film of the abdomen showed the presence of fluid levels in the gut. Exploration was undertaken forthwith.

The appendix was found to be acutely inflamed and perforated in several places. In the wall of caecum at its base was a small hard white lump. This was removed along with the appendix, a rubber caecostomy tube being inserted to fill the gap. Section through the caecal wall at the base of the appendix showed diffuse infiltration of the muscular coat by trabeculae of carcinoma cells.

Case 3. A very frail old lady of 65 years was admitted with a vague recent history of recurring bouts of abdominal colic and of occasional diarrhoea. For ten days or so she had been conscious of a tender lump in the right lower abdomen.

Her temperature on admission was $100^{\circ}$ and there was a smooth, rounded, very tender, fixed mass in the right iliac fossa-the size of a large orange.

A provisional diagnosis of appendicitis with abscess formation was made and conservative treatment advised. When, after six days, there being no improvement, the abdomen was opened, the hard lump was proved to be a caecal cancer. No obvious abscess was discovered but the appendix itself was not identified.

Case 4. A thin, pale boot repairer, aged 44, had complained of recurring spasms of abdominal colic of three weeks' duration, and also of increas- ing anorexia, tiredness and pallor. There was an obvious tender lump in the right lower abdomen; his leucocyte count was 24,500 per c.mm. and his haemoglobin 44 per cent. A provisional diagnosis of ' appendix abscess' was made, and at operation an abscess cavity was found on the anterior aspect of the caecum, which was suspiciously indurated and fixed to th $\geqslant$ posterior abdominal wall.

The abscess was drained and later a staged and difficult right hemi-colectomy was carried out, when the true diagnosis of caecal cancer was confirmed.

(c) The patient in whom the presence of infection confuses and confounds the diagnosis.

Case 5. A thin and rather tired looking young man of 37 years was admitted to hospital as an emergency, complaining of recurring spasms of lower abdominal colic of eight days' duration, accompanied by constipation and some vomiting.

His abdomen was uniformly distended but no tumour could be felt and there was no localized area of tenderness.

By the following morning his temperature was $99^{\circ}$ and his pulse 100 . There was now marked abdominal tenderness, especially on the right side. It was thought possible that he had acute appendicitis, a new growth complicated by infection or even a caecal amoeboma.

At laparotomy a small collection of pus was found lying close to the appendix, which was as thick as one's ring finger but not obviously perforated. When traced upwards it could be seen disappearing into a hard irregular tumour involving the posterior wall of the caecum. This was later proved to be an adenocarcinoma to which coils of ileum were bound by granulation tissue containing chronic inflammatory cells, foreign body giant cells and many polymorphs.

Case 6. A housewife, aged 60 years, first attended in April r 938 complaining of occasional attacks of abdominal pain, three only in the previous five months. She was seen only once in the out-patient department when a hard, nodular and slightly tender lump was felt low down in the right iliac fossa. She failed to attend again until September. In the meantime she had had three more attacks of pain, but the abdominal tumour was now no bigger than a pigeon's egg. It was, however, still tender and inguinal adenitis was suggested as the most likely diagnosis. Surgical advice was now sought when the alternative diagnoses of tuberculous adenitis or of strangulated omentocele were suggested.

In October the swelling was explored when about ${ }_{15} \mathrm{ml}$. of thick pus was evacuated from an intraperitoneal abscess cavity, in the depths of which a hard lump could be felt lying on the iliacus muscle, but its true nature was not estab- 
lished at this stage. Later, however, a barium enema revealed a persisting filling defect in the caecum and at a subsequent operation a caecal cancer was resected. A large pericaecal abscess was discovered which was packed with iodoform gauze and the patient made an uninterrupted recovery. Section of the tumour showed it to be a well-differentiated adenocarcinoma.

(d) The patient with acute appendicitis diagnosed clinically, confirmed at operation and later firmly established by histological section, which turns out ultimately to be no more than the clinically apparent infective manifestation of an underlying cancer.

The surgeon is content to find his pre-operative diagnosis of acute appendicitis confirmed and if he had any doubts about the diagnosis before, they are now quite dispelled when he retrieves an obviously inflamed and often perforated appendix. He may perchance find an area of induration in the caecum but this, if he is unwary, he may confidently dismiss as part and parcel of the acute appendicular lesion. Recovery may be without incident and healing complete in the normal time. All is now well for months or maybe even years, until there show themselves, and often in an intractable form, the all too familiar symptoms such as anorexia, asthenia, anaemia and the like, which we have come to associate with malignant disease of the proximal colon. The time relationship between the two episodes may be so close as to leave little doubt in the mind of the surgeon, or so long as to leave the connection between the two a matter of speculation alone.

This type of presentation is illustrated by the following clinical records.

Case 7. An old man of 73 years was admitted to hospital complaining of recurring attacks of colic in the right lower abdomen during the previous 36 hours. His general condition was good and his temperature was only $99^{\circ}$. Just medial to the right anterior superior spine was a tender mass which seemed to be lying close under the skin. A diagnosis of acute appendicitis was made and on exploration a grossly swollen appendix was found lying doubled up immediately medial to the caecum. It was perforated at its base. On palpation of the caecum through its wall, near the base of the appendix there could be felt what was taken to be a hard malignant ulcer, the size of a threepenny piece. There were no obviously enlarged lymph glands nearby. The appendix was removed along with a small cuff of the caecum containing the growth, but section of the specimen was reported to show no cancerous tissue. He made a good recovery and was discharged with a diagnosis of acute appendicitis.

Three months later he returned complaining of lower abdominal pain and diarrhoea. There was now an obvious hard fixed mass in the right iliac $\frac{3}{0}$ fossa, and visible peristalsis. A clinical diagnosis $\cong$ of caecal cancer was confirmed at laparotomy when an abscess cavity was also encountered lying medial to the ascending colon.

The first pre-operative diagnosis of acute appendicitis was here confirmed at operation. A firm suspicion of associated malignant disease was not established histologically (the section has since been re-examined) and the quick and apparently complete recovery was allowed to allay all suspicion.

Case 8. This patient first came into hospital in June 1936 at the age of 55 years. She gave a three days' history of abdominal pain, first at the umbilicus and later settling into the right iliac fossa. Her temperature was $102^{\circ}$ and a tender lump, the size of an orange, could be felt just medial to the right anterior superior spine. A diagnosis of appendicitis with early abscess formation was made but operation was delayed for two days, when a perforated gangrenous appendix was removed.

She was re-admitted in November 1939 with a two months' history of griping abdominal pain and diarrhoea. She was now found to have a firmo nodule in the aptendix scar and a more deeple situated mobile lump. At laparotomy an in operable carcinoma of the caecum was found with extension intc the muscles of the abdominal wall.

Although we have in this instance no exact proof of the existence of the cancer already at the first operation $3 \frac{1}{2}$ years before, the findings of a nodule in the appendix scar at the second certainly suggested the likelihood of this being so.

(e) Not very dissimilar from those in the previous group, there are the patients in whom a pre-operative diagnosis of appendix mass or of appendix abscess is made, a diagnosis which appears to be confirmed at operation when the associated carcinoma is overlooked.

Case 9. This patient, who was described in her notes as a ' plump, jolly old woman of 7I,' was admitted urgently to hospital with a two weeks' history of colicky lower abdominal pain and anorexia. Her temperature was $100^{\circ}$ and there was a fixed tender mass in the right iliac fossa. A provisional diagnosis of appendicitis with abscess formation was made, with pericaecal abscess from a carcinoma as a less likely alternative. She was first treated expectantly but seven days later, because of persisting pain and a swinging temperature, the abscess was incised and drained. She was discharged a month later with a diagnosis of ' appendix abscess,' her operation incision being at this date still unhealed.

She returned, however, two months later, ' wasted and unhappy.' There was no doubt now 
about the large tender fixed mass in the right lower abdomen and there was a recent discharge of pus and of blood clot from a sinus which had developed in the operation scar. She died on the following day and autopsy confirmed the presence of a caecal cancer with pericaecal inflammation extending distally into the muscles of the thigh.

It is easy enough to understand how the tumour in such cases can be missed at operation, and that for a variety of reasons:-

(I) The finding of an abscess cavity in the region of the appendix seems to confirm the pre-operative diagnosis and makes one forget to search further for some other explanation.

(2) The presence of any area of hardness in the region of the caecum seems reasonably to be explained by its close proximity to the abscess cavity.

(3) The presence of an abscess makes it both difficult and unwise to carry out a really wide and careful exploration.

The inflammatory appendix mass, consisting as it does of the inflamed appendix concealed and buttressed by adherent omentum and coils of small gut, commonly resolves without incident under purely conservative treatment. Even the early abscess may clear spontaneously, emptying itself, maybe, into the bowel or into the rectum. When things go wrong, and this is especially so in an older person, when increasing symptoms and extending signs point to a progressing infection, it is wise always to consider the possibility of an underlying cancer as the primary pathology. This is especially so when confronted with a patient who looks altogether too thin, too pale and too frail to be explained away by what seems to be only a short infective illness.

(f) Persisting discharge from an appendicectomy wound may be due to a variety of causes. It may point to no more than a residual and inadequately drained abscess, or to an appendix stump which has been left behind or which harbours a faecolith. The discharge in either event tends after a time to dry up without treatment, as will a faecal leak which follows the separation of a sloughing patch on the caecal wall. As time passes and there is still no sign of healing and no revealing rejection of silk or cotton thread, we think of faulty swab counts, of tuberculosis, of actinomycosis, or of regional ileitis. It would be wise at this stage to bear in mind in the young, as in the old, the possibility of an underlying ceacal cancer, especially if there is a history before the recent acute inflammatory episode of abdominal colic, diarrhoea, anorexia, anaemia and the like.

Careful palpation, if necessary under general anaesthesia, may sometimes reveal an underlying lump, but this finding might equally support almost any of tbe alternative diagnoses. Indura- tion of the sinus tracks, appreciated on probing, to the accompaniment of bleeding, is no more specific. The really helpful investigations are a barium enema, which may show clearly a filling defect in the bowel at the site of the sinus, or biopsy of the wound track, always provided it is carefully taken from the depths of the wound. It is as true of this condition as it is of any biopsy taken elsewhere, that it must be adequate in size, carefully selected and as representative as possible, and a negative answer must never be allowed to blunt the acuteness of the surgeon's critical alertness.

That a cancer may be unsuspected before operation and overlooked even when the abdomen is opened and the appendix removed and, further, that this possibility may first be seriously entertained only when the tumour grimly erupts on the surface-this is a happening of which I am painfully aware and that I am sure many honest surgeons will admit to sharing just such an experience.

In the following cases, both treated in our hospital, the diagnosis was established when it had become only clearly apparent as the tumour fungated out along the appendicectomy wound.

Case ro. The patient, a young man of 44 years, was admitted urgently to hospital with a recent history of recurring attacks of fairly generalized abdominal colic. Two days prior to admission this had become much more severe and had later settled down in the right lower abdomen.

A tender lump was felt in the right lower abdomen. This was believed to be an appendix mass and expectant treatment was advised. Next day, however, because of signs of spreading infection the abdomen was opened. The appendix, with a perforation at its base, was found lying in an abscess cavity. Its removal was tedious and difficult, and during the dissection a portion of the rather necrotic caecal wall was torn. This was dealt with by exteriorization of the caecum. His convalescence was punctuated by recurring attacks of colic and prolonged by digestion of the skin edges. Almost three weeks later an extraperitoneal closure of the caecostomy was done, but difficulty was encountered in dealing with what was described as a hard, inflamed and oedematous bowel. Healing was eventually complete and he was allowed home.

He soon returned, however, complaining now of the passage of blood and faeces from a re-opened caecal fistula. In the middle of the old operation scar was a large irregular sinus ringed by an extensive collar of hard, nodular growth raised a little above the level of the surrounding skin (Fig. 4). This tissue bled on the slightest pressure and was found to be only the superficial limit of a 
hard, fixed mass which could be felt extending backwards and laterally in the right iliac fossa. It was now clear that we had overlooked from the start a malignant growth in the colon, a diagnosis which was at once confirmed when biopsy from the edge of the fistula revealed anaplastic spheroidal cell carcinoma extending up to and involving the skin (Fig. 5).

Case I I. This 56-year-old clerk first began to complain of diarrhoea early in $\mathrm{r} 94 \mathrm{I}$; this was followed later that year by constant ' nagging' abdominal pain. In April 1942 he was admitted to hospital where drainage was undertaken for what was taken to be an appendix abscess. Following the operation he developed a faecal fistula in the wound which refused to heal. Its margins were cauterized on two occasions without benefit, and when in June a biopsy was taken it showed no more than an inflammatory reaction.

He first came to Hammersmith Hospital in November 1942 , by which time he had lost 3 stone in weight in the previous 18 months. He was thin and pale and a large mass could be felt largely filling the right lower abdomen. It was tender, firm, irregular and fixed. Crossing its lateral onethird was an unhealed McBurney incision which was gaping and discharging. Biopsy taken from the somewhat everted medial margin showed the presence of a mucus-secreting adenocarcinoma involving skin.

How does the presence of pericaecal inflammation or of a complicating appendicitis modify treatment?

Formal right hemicolectomy in the presence of an acute episode of inflammation would be to invite the risk of the gravest infection. It is wise to limit the first operation to simple drainage of the $\stackrel{\mathbb{Q}}{\&}$ abscess through a short muscle cutting incision $\stackrel{c}{c}$ made over the summit of the abscess and as far $\Rightarrow$ laterally as its situation will allow. It may be $\stackrel{\mathscr{S}}{\rightarrow}$ allowable in some instances to do at the same time (and preferably as the first step in the operation) an ileo-transverse colostomy, through a paramedian incision, especially if there is any evidence of obstruction.

It is only necessary to wait until the acute episode has subsided - in the average case this will take Io to I5 days - and then to proceed with a formal resection. A long oblique muscle cutting incision is now made passing on either side of the drainage wound to include all of the infected tissue of the abdominal wall. Apart from the varying difficulty in the dissection, the rest of the resection follows the usual lines.

\section{Summary}

In a high proportion of patients with cancer of the caecum and ascending colon there are inflammatory complications of which pericolic abscess and acute appendicitis are the most frequent. This accident often leads to errors in clinical diagnosis and even at operation the primaro cancer may only too easily be overlooked. Some. of the difficulties encountered in the diagnosis: and management of these patients are underlined by illustrative case records.

The photographs are the work of Mr. J. V. Willmott and his staff.

\section{BIBLIOGRAPHY}

BARTLETT, M. K., and MILLER, R. H. (1940), New England F. Med., 222, 783 .

BROWN, C. H., COLVERT, J. R., and BRUSH, B. E. (1948), Ann. Int. Med., 28, 940.
MAYO, C. W. (1941), Proc. Staff Meet. Mayo Clinic, 16, 67. WAKELEY, C. P. G., and RUTHERFORD, R. (1932), Brit. $₹$. Surg., 20, 91. 